\title{
Pre-cART Elevation of CRP and CD4+ T-cell Immune Activation Associated with HIV Clinical Progression in a Multinational Case-Cohort Study
}

\author{
Ashwin Balagopal, M.D. ${ }^{1}$, David M. Asmuth, M.D. ${ }^{2}$, Wei-Teng Yang, M.D. ${ }^{1}$, Thomas B. \\ Campbell, M.D. ${ }^{3}$, Nikhil Gupte, Ph.D. ${ }^{1}$, Laura Smeaton, M.S. ${ }^{4}$, Cecilia Kanyama, M.D. ${ }^{5}$, \\ Beatriz Grinsztejn, M.D., Ph.D. ${ }^{6}$, Breno Santos, M.D. ${ }^{7}$, Khuanchai Supparatpinyo, M.D. ${ }^{8}$, \\ Sharlaa Badal-Faesen, M.B.B.Ch. ${ }^{9}$, Javier R. Lama, M.D., M.P.H. ${ }^{10}$, Umesh G. Lalloo, \\ M.B.Ch.B., M.D. ${ }^{11}$, Fatima Zulu, M.Sc. ${ }^{12}$, Jyoti S Pawar, M.B.B.S. ${ }^{13}$, Cynthia Riviere, M.D. ${ }^{14}$, \\ Nagalingeswaran Kumarasamy, M.D. ${ }^{15}$, James Hakim, M.B.Ch.B. ${ }^{16}$, Xiao-Dong Li, M.D., \\ Ph.D. ${ }^{17}$, Richard B. Pollard, M.D. ${ }^{17}$, Richard D. Semba, M.D., M.P.H. ${ }^{18}$, David L. Thomas, \\ M.D., M.P.H. ${ }^{1}$, Robert C. Bollinger, M.D. ${ }^{1}$, Amita Gupta, M.D. ${ }^{1}$, and the ACTG PE ARLS and \\ NWCS 319 Study team
}

${ }^{1}$ Department of Medicine, Johns Hopkins University School of Medicine, Baltimore, MD, US ${ }^{2}$ Department of Medicine, University of California Davis, Sacramento, CA, US ${ }^{3}$ University of Colorado Denver, Aurora, CO, US ${ }^{4}$ Harvard School of Public Health, Boston, MA, US ${ }^{5}$ University of North Carolina Project, Lilongwe, Malawi ${ }^{6}$ Instituto de Pesquisa Clinica Evandro Chagas, Manguinhos, Brazil ${ }^{7}$ Hospital Nossa Senhora da Conceição, Porto Alegre, Brazil ${ }^{8}$ Chiang Mai University, Chiang Mai, Thailand ${ }^{9}$ Department of Medicine, University of Witwatersrand, Johannesburg, South Africa ${ }^{10}$ IMPACTA PERU Clinical Trials Unit, Asociacion Civil Impacta Salud y Educacion, Lima, Peru ${ }^{11}$ University of KwaZulu Natal, Nelson R Mandela School of Medicine, Durban, South Africa ${ }^{12}$ Malawi College of Medicine-Johns Hopkins Research Project, Kachere Rehabilitation Centre, Blantyre, Malawi ${ }^{13}$ National AIDS Research Institute, Pune, India ${ }^{14}$ Les Centres GHESKIO, Port-au-Prince, Haïti ${ }^{15}$ YR Gaitonde Center for AIDS Research and Education, Chennai, India ${ }^{16}$ University of Zimbabwe, Harare, Zimbabwe ${ }^{17}$ Department of Medicine, University of California Davis, Sacramento, CA, US ${ }^{18}$ Department of Ophthalmology, Johns Hopkins University, Baltimore, MD, US

\section{Abstract}

\begin{abstract}
Background-Despite the success of combination antiretroviral therapy (cART), a subset of HIV-infected patients who initiate cART develop early clinical progression to AIDS; therefore some cART initiators are not fully benefitted by cART. Immune activation pre-cART may predict clinical progression in cART initiators.
\end{abstract}

\footnotetext{
Corresponding author: Ashwin Balagopal, MD, 855 N. Wolfe Street, Rangos Rm. 535, Baltimore, MD, 21025, Tele: 1-443-286-7666, abalago1@jhmi.edu; Alternate corresponding author: Amita Gupta, MD MHS, 600 N. Wolfe Street, Phipps 540B, Baltimore, MD 21231, Tele: 1-410-502-7696, agupta25@jhmi.edu.

Conflicts of Interest. The authors declare no personal or professional conflicts of interest.

Presentations. Portions of this manuscript have been previously presented at the $19^{\text {th }}$ and $20^{\text {th }}$ Conference on Retroviruses and Opportunistic Infections in 2012 (D-192) and 2013 (R-271).
} 
Methods-A case-cohort study ( $\mathrm{n}=470$ ) within the multinational Prospective Evaluation of Antiretrovirals in Resource-Limited Settings (PEARLS) clinical trial (1571 HIV treatment-naïve adults who initiated cART; CD4+ T cell count $<300$ cells $/ \mathrm{mm}^{3}$; nine countries) was conducted. A subcohort of 30 participants/country was randomly selected; additional cases were added from the main cohort. Cases ( $\mathrm{n}=236$ [random subcohort-36; main cohort-200]) had clinical progression (incident WHO Stage 3/4 event or death) within 96 weeks following cART initiation. Immune activation biomarkers were quantified pre-cART. Associations between biomarkers and clinical progression were examined using weighted multivariable Cox-proportional hazards models.

Results-Median age was 35 years, $45 \%$ were women, $49 \%$ black, $31 \%$ Asian, and $9 \%$ white. Median CD4+ T-cell count was 167 cells $/ \mathrm{mm}^{3}$. In multivariate analysis, highest quartile CRP concentration (adjusted hazards ratio [aHR] 2.53, 95\% CI 1.02-6.28) and CD4+ T-cell activation (aHR 5.18, 95CI 1.09-24.47) were associated with primary outcomes, compared to lowest quartiles. sCD14 had a trend towards association with clinical failure (aHR 2.24, 95\% CI 0.965.21).

Conclusions-Measuring CRP and CD4+ T-cell activation may identify patients with CD4+ T cell counts $<300$ cells $/ \mathrm{mm}^{3}$ at risk for early clinical progression when initiating cART. Additional vigilance and symptom-based screening may be required in this subset of patients even after beginning cART.

\section{Keywords}

immune activation; global HIV; cART clinical outcomes; C-Reactive protein; T-cell activation

\section{Introduction}

HIV infects more than 30 million people worldwide, but in most people clinical progression has been controlled with combination antiretroviral therapy (cART). Some patients, however, remain at risk for early progression and death even after starting cART, suggesting that cART is only partially effective at controlling progression in these patients ${ }^{1-5}$. Furthermore, morbidity and mortality rates among persons from resource-limited settings exceed those in resource-rich settings, most significantly during the first year after cART initiation ${ }^{6-9}$. A subset of treated patients who remains high risk for early clinical HIV progression may require more frequent and closer follow-up than is the current standard of care ${ }^{10}$. Finding baseline factors that identify such high-risk patients across multiple geographic and socioeconomic settings could optimize clinical management strategies, as well as identify common pathogenic mechanisms driving HIV disease progression ${ }^{11}$. A number of factors have been associated with clinical response to cART, including pre-cART clinical stage, CD4+ T-cell count, and HIV viral load. However, the role of baseline inflammation has not been well evaluated, particularly in low- and middle-income settings $12-16$.

Chronic immune activation is a hallmark of HIV infection that has been associated with viral replication, CD4+ T-cell depletion, and other dysfunctions of the immune system ${ }^{17}$. Although the causes of immune activation in HIV infection remain unclear, previous studies have associated baseline elevations of a number of immune-activation biomarkers with 
clinical HIV progression among patients initiating cART, including TNF-a, IL-6, IFN- $\gamma$, Tcell activation, sCD14 and other microbial translocation (MT) markers ${ }^{18-22}$. However, these studies have only partially accounted for underlying factors that might contribute to immune activation and were conducted in resource-rich communities or in single-site settings. In addition, the findings from these studies have not been consistently replicated ${ }^{23}$. Thus, it is unclear if there are biomarkers that would be universally predictive of clinical response following cART initiation that are independent of geographic location, resource abundance, and diversity of endemic diseases.

Therefore, we conducted a case-cohort analysis of the AIDS Clinical Trials Group (ACTG) Prospective Evaluation of Antiretrovirals in Resource-Limited Settings (PEARLS) A5175 study, a clinical trial of antiretroviral therapy among HIV-infected, cART-naive adults from nine countries ${ }^{24}$. The diversity of our cohort allowed for assessment of a comprehensive panel of soluble and cellular immune-activation biomarkers to identify baseline predictors of HIV clinical progression following cART initiation that are independent of geographic setting.

\section{Methods}

\section{Study Design and Population}

The methods and procedures of the ACTG PEARLS A5175 study (ClinicalTrials.gov NCT00084136), a Phase IV randomized controlled clinical trial of three antiretroviral therapy regimens among 1571 treatment-naïve adults from nine countries (Brazil, Haiti, India, Malawi, Peru, South Africa, Thailand, the USA, and Zimbabwe), have been previously described in detail ${ }^{24}$. In brief, the study was conducted between May 2005 and August 2007. Study inclusion criteria were CD4+ T cell count $<300$ cells $/ \mathrm{mm}^{3}$ and no recent acute illness (i.e. pneumonia, gastroenteritis, or pelvic inflammatory disease) or opportunistic infections. Participants were randomized to receive lamivudine-zidovudine + efavirenz, didanosine + emtricitabine + atazanavir, or tenofovir-emtricitabine + efavirenz, and median follow-up was 142 weeks. Viral resistance testing was not available at baseline. The primary outcome was time from treatment randomization to HIV disease progression. Informed consent, including permission to use biological materials, was obtained from all participants, and the human experimentation guidelines of the US Department of Health and Human Services and local site institutional review boards and ethics committees were followed.

A case-cohort ${ }^{2526}$ of 470 participants comprising a randomly selected subcohort $(n=270)$ was chosen from the parent cohort, to which were added additional cases $(n=200)$ selected non-randomly from the parent cohort: a) the random subcohort component allows estimates disease prevalence and immune-activation biomarker distributions among the parent cohort; b) the addition of cases from the parent cohort allows adequate power to determine the association of individual biomarkers with the primary outcome, defined as incident WHO Stage 3 or 4 event or death by 96 weeks after cART initiation. The randomly selected subcohort of 270 parent study participants (30 per country) included 36 individuals meeting our primary outcome (Figure 1; Table 1). Immune activation biomarkers were quantified among the full case-cohort using pre-cART serum, plasma and peripheral blood 
mononuclear cell (PBMC) samples. Serum and plasma were stored in a centralized $-80^{\circ} \mathrm{C}$ repository until the time of testing. Viable pre-cART PBMC were collected, cryopreserved and stored in liquid nitrogen. Three markers of microbial translocation (MT) were measured, including gram-negative bacterial lipopolysaccharide [LPS], sCD14, and EndoCAb IgM. Five soluble inflammatory cytokines were measured, including C-reactive protein [CRP], IP-10, TNF-a, IL-6, and IFN- $\gamma$. Two T-cell activation phenotypes (CD4+/DR+/CD38+ and CD8+/DR+/CD38+) were also measured.

Laboratory Testing-To minimize laboratory-to-laboratory variation and standardize testing across sites, single laboratories performed batch testing for each marker. LPS, sCD14, and EndoCAb IgM, were measured in one laboratory at Johns Hopkins University. LPS was quantified using an optimized Limulus Amebocyte Lysate (LAL) assay (Lonza, Walkersville, MD) ${ }^{27}$. Commercially available ELISA kits were used for measurement of sCD14 (R\&D Systems, Inc., Minneapolis, MN), EndoCAb IgM (Cell Sciences, Canton, MA), and CRP (CRP Quantikine ELISA, R\&D Systems, Minneapolis, MN). The remaining biomarkers were tested in an externally quality-assured ACTG-designated laboratory at the University of California Davis. IP-10 (IFN- $\gamma$ inducible protein-10, CXCL10) was measured using commercially available testing kits (electrochemiluminescent bridging immune-assay by Meso-Scale Discovery, Gaithersburg, Maryland, USA). Soluble TNF-a, IL-6, and IFN- $\gamma$ were measured using the Luminex multiplex cytokine platform (R\&D Systems, Inc., Minneapolis, MN). Viable pre-cART PBMC samples from 157 participants were available for testing and were stained with conjugated antibodies to CD4, CD8, HLA-DR and CD38 (BD BioSciences, San Jose, CA). T-cell activation (CD4+ and CD8+) was measured by quantifying the percentages of HLA-DR+/CD38+ cells using flow cytometry.

\section{Statistical Analyses}

To determine whether the random subcohort was representative of the full parent cohort, Wilcoxon Rank Sum and Chi-Square tests were used to compare continuous and categorical covariates, respectively. All biomarkers were parameterized in quartiles to accommodate right- or left-censored data, except for LPS, which, despite using an optimized assay ${ }^{27}$, was only detected in samples from 137/367 (37\%) participants and was subsequently dichotomized as detectable vs. undetectable (Table 2). Kruskal-Wallis non-parametric tests were used to compare pre-cART biomarker values among baseline demographic and biologic parameters, and inverse probability weighting was used to account for the casecohort study design in identifying baseline covariates associated with each biomarker. Univariate and multivariate Cox-proportional hazards models weighted for case-cohort sampling were used to identify pre-cART biomarker values (grouped by quartile) that predicted the primary outcome. Multivariate models were stratified for country and treatment group and adjusted for baseline age, sex, body mass index (BMI), CD4+ T-cell count, and hemoglobin. Because baseline HIV RNA was largely collinear with CD4+ T cell count and hemoglobin, adjustment for baseline HIV RNA level did not substantially contribute to a model in which the latter two covariates were included. Albumin was collinear with hemoglobin, and so was not added to the models. A separate model also adjusted for baseline tuberculosis (TB). Receiver operator curve (ROC) analysis was 
performed to estimate the C-statistic and the corresponding 95\% CI for CRP at each cutoff, before and after adjusting for covariates.

\section{Results}

\section{Baseline Characteristics}

The random subcohort was not statistically different from the parent cohort by baseline demographics, clinical status, and laboratory results, except for a slightly lower baseline creatinine clearance although all participants had $\mathrm{CrCl} \varangle 60 \mathrm{cc} / \mathrm{min}(\mathrm{CrCl}<60 \mathrm{cc} / \mathrm{min}$ was an exclusion criterion for the parent study). ${ }^{24}$ The case-cohort had a median age (interquartile range [IQR]) of 35 years (29-40) and was composed of 215 (45\%) women and 231 (49\%) black, 146 (31\%) Asian, and 48 (9\%) white individuals (Table 1). The median BMI (Interquartile Range [IQR]) was $21.8 \mathrm{~kg} / \mathrm{m}^{2}$ (19.6-24.4), with 97 (21\%) overweight or obese and $58(12 \%)$ undernourished or underweight participants. On enrollment, the median CD4+ T-cell count (IQR) was 167 cells $/ \mathrm{mm}^{3}$ (79-219), and the median plasma HIV RNA level (IQR) was $5.11 \log _{10} \mathrm{cp} / \mathrm{mL}$ (4.60-5.53). TB co-infection was common (24\%), and the prevalence of hepatitis B virus co-infection was $5 \%$.

In the parent trial of 1571, $236(15 \%)$ participants were cases who developed the composite primary outcome of WHO Stage 3 or 4 diagnosis or death by 96 weeks after cART initiation. The most frequent incident events were TB (27.1\%), death (16.9\%), cytopenia (15.7\%), serious bacterial infection (11.4\%), and weight loss (10.6\%) (SFig. 1). Notably, cases from Haiti, India, and Thailand were disproportionately more likely to experience weight loss than cases from other countries, and TB was one of the top three incident diagnoses among cases from all countries, except the USA (data not shown). Cases differed from controls by race, country, BMI, and baseline CD4+ T-cell count ( $<<0.01)^{24,28}$, and cases were more likely to have a history of past or current TB $(\mathrm{p}=0.001)$ (Table 1).

\section{Pre-cART Immune Activation}

All available blood, plasma and PBMC samples were used to quantify biomarkers. Notably, some of the blood and plasma samples from India could not be exported, and participants from Thailand and India did not have archived PBMC samples. Compared to participants lacking PBMC samples, those with available samples for T-cell activation measurement had similar baseline age, gender, BMI, hemoglobin, and CD4+ T-cell count but a significantly lower baseline median HIV RNA level (4.99 $\log _{10} \mathrm{cp} / \mathrm{mL}$ vs. $5.18 \log _{10} \mathrm{cp} / \mathrm{mL}, \mathrm{p}=0.04$ ). Table 2 shows the median pre-cART biomarker values grouped by quartile in the random subcohort and by study group in the full case-cohort. Except for CRP and IP-10, pre-cART biomarker values did not differ significantly between cases and controls. Compared to controls, cases had significantly higher median concentrations of CRP ( 6.3 vs. $3.03 \mathrm{mg} / \mathrm{mL}$, $\mathrm{p}=0.02$ ) and IP-10 (2079.11 vs. $1203.81 \mathrm{pg} / \mathrm{mL}, \mathrm{p}=0.001$ ). Individual pre-cART immuneactivation biomarkers were associated with a number of baseline study population characteristics. Notably, except for CRP $(\mathrm{p}=0.10)$ and $\mathrm{CD} 8+\mathrm{T}$-cell activation $(\mathrm{p}=0.11$; data not shown), biomarkers varied significantly by country ( $\mathrm{p}<0.001$; SFig. 2 ). 


\section{Predictors of HIV Clinical Progression on CART}

Cox-proportional hazards models stratified for country and treatment group were used to estimate the hazards of the primary outcome (incident WHO stage 3 or 4 disease or death within 96 weeks after cART initiation), for each pre-cART biomarker level grouped by quartile relative to the lowest quartile. In univariate analysis, elevated baseline levels of several biomarkers (highest quartile relative to lowest quartile) were associated with clinical progression following cART initiation, including sCD14 (Hazard Ratio [HR] 2.20, 95\% CI 1.01-4.79), IP-10 (HR 2.81, 95\% CI 1.27-6.24), CRP (HR 2.19, 95\% CI 1.11-4.34), CD4+/DR+/CD38+ (HR 4.39, 95\% CI 1.22-15.69), and CD8+/DR+/CD38+ (HR 6.32, 95\% CI 1.50-26.67) (Table 3). In multivariate analyses adjusting for baseline sex, age, BMI, CD4+ T-cell count, and hemoglobin, only highest quartile CRP (adjusted HR [aHR] 2.53, 95\% CI 1.02-6.28) and CD4+/DR+/CD38+ T-cell activation (aHR 5.18, 95\% CI 1.09-24.47) remained associated with the primary outcome, compared to the first quartile of both markers (Table 3 and SFig. 3). The highest quartile of sCD14 showed a trend toward an association with clinical progression (aHR 2.24, 95\% CI 0.96-5.21), as did the highest quartile of CD8+//DR+/CD38+ T-cell activation (aHR 4.28, 95\% CI 0.88-20.76). Adjustment for baseline HIV RNA level did not substantially alter the models (Table 3 footnote). To account for the high pre-cART TB prevalence, separate multivariate models were developed that adjusted for TB at study entry and showed similar findings: there was a trend towards association that does not reach statistical significance for the highest quartile CRP (aHR 2.43, 95\% CI 0.98-6.04; $\mathrm{p}=0.06$ ) and clinical progression, whereas CD4+/DR+/ CD38+ T-cell activation maintained its association with clinical progression (aHR 5.56, 95\% CI 1.04-29.76; $\mathrm{p}=0.045$ ) (Supplementary Table). In addition, when we set a strict definition of incident TB as occurring $>4$ weeks after entry to avoid misclassifying persons with subclinical prevalent TB as having incident TB, we still note that persons with the highest quartile CRP values at baseline have an increased HR of $1.63(\mathrm{p}<0.05)$ of developing a WHO Stage 3 or 4 outcome.

We explored Receiver Operating Characteristic (ROC) curve analysis to assess the predictive power of pre-cART CRP concentration as a marker for clinical HIV progression post cART initiation (i.e. incident WHO Stage 3 or 4 disease or death by 96 weeks on cART). Highest quartile CRP concentration (>10 mg/L) had a specificity of $77 \%$ but low sensitivity (40\%). Lowering the cutoff to CRP concentration $>5 \mathrm{mg} / \mathrm{L}$ had the best predictive power but overall low sensitivity (55\%) and specificity (62\%). In unadjusted ROC curve analysis, the area under the curve (AUC) for CRP greater than $5 \mathrm{mg} / \mathrm{L}$ was 0.60 . After adjusting for baseline age, sex, country, BMI, hemoglobin, CD4+ T-cell count, plasma HIV RNA, and baseline TB, the AUC was 0.71, irrespective of the cutoff used (CRP>5 $\mathrm{mg} / \mathrm{L}$ or $\mathrm{CRP}>10 \mathrm{mg} / \mathrm{L}$; Figure 2), indicating that CRP levels pre-cART might have fair discriminating power to distinguish HIV-infected persons who are likely to clinically progress after cART initiation from persons who are unlikely to progress.

\section{Discussion}

To our knowledge, this is the first study to assess and identify baseline biomarkers associated with early clinical HIV progression following cART initiation across multiple 
resource and clinical settings. After measuring a comprehensive panel of immune-activation biomarkers, our analysis indicates that only the highest quartiles of baseline pre-cART CRP concentration and CD4+ T-cell activation predict subsequent progression to WHO stage 3 or 4 disease or death within 96 weeks on cART after adjusting for baseline age, sex, BMI, and CD4+ T-cell count. Notably, the associations were independent of country-to-country differences in baseline biomarker levels and cART regimen. Baseline high CRP levels and CD4+ T-cell activation also remained associated with clinical progression. Conversely, while a trend towards significant association was observed for SCD14 and CD8+ T-cell activation, we could not confirm an association between early HIV progression on cART and biomarkers that have been previously described in relation to HIV disease progression, namely in LPS, EndoCAb IgM, IL-6, TNF-a, and IP-10 17,29-31. In resource-limited settings where follow-up intervals can be prolonged, our findings may help clinicians identify highrisk cART-naïve patients who require additional vigilance for OIs and AIDS progression even after cART initiation.

The response to HIV treatment can vary considerably and AIDS-related events can still develop in the early months after cART initiation ${ }^{10,32}$. Indeed, AIDS-related events are still the leading cause of early mortality among persons living with HIV on CART in low- and middle-income settings, even among those with virologic suppression ${ }^{15,33}$. Consequently, current treatment guidelines offer no provision for patients who start cART but who will ultimately clinically progress. A baseline prognostic marker would be of particular importance in such settings, where tests for HIV RNA level and CD4+ T cell counts tests may be prohibitively expensive 6,11 .

Immune activation has been previously described in HIV-infected persons, but has been defined using differing biomarker panels, and most prior studies have been conducted at a single site or in resource-rich countries. In resource-limited settings, high CRP levels among untreated HIV-infected women who were pregnant has been previously associated with progression to WHO stage 4 disease or death in the mothers and in their children ${ }^{34}$. In addition, elevated CRP levels were associated with clinical progression in an HIV-infected cART-naive population of Ugandans ${ }^{35}$. More recently, the SMART and INSIGHT studies have associated elevated pre-cART CRP levels with death, opportunistic infection, and AIDS progression among patients in resource-rich countries ${ }^{36,37}$. To our knowledge, however, these are the first data to show that pre-cART CRP levels are associated with clinical progression in HIV-infected persons initiating cART in settings with mixed resource levels. In contrast, the evidence regarding CD4+ T-cell activation has been less consistent. Apart from the present analysis, only one other study has associated baseline CD4+ T-cell activation with outcomes among patients initiating $\mathrm{cART}^{38}$, whereas several studies have found no such association $37,39,40$. Thus, our analysis supports and expands previous CRP findings in resource-rich settings now to diverse resource-limited settings while also adding evidence linking CD4+ T-cell activation to cART outcomes.

CRP assays are relatively inexpensive and could be utilized in resource-limited and resource-rich settings. Our finding that elevated baseline CRP concentration greater than $10 \mathrm{mg} / \mathrm{L}$ is independently associated with poor clinical response to cART may provide clinicians in multiple settings with a more generalizable approach to risk-stratify patients 
prior to cART initiation. Furthermore, despite its lack of specificity, CRP (an acute phase reactant produced by the liver in response to general inflammatory stimuli) has remarkable predictive value in varied conditions from cardiovascular disease to osteomyelitis ${ }^{41,42}$. For example, among healthy persons, a CRP concentration greater than $3 \mathrm{mg} / \mathrm{L}$ is an independent risk factor for cardiovascular risk ${ }^{43}$. In our study, cases had a median baseline $\mathrm{CRP}$ of $6 \mathrm{mg} / \mathrm{L}$ compared to that of $3 \mathrm{mg} / \mathrm{L}$ in controls, supporting its potential clinical utility to distinguish HIV-infected persons who may progress after cART initiation. However, larger cohort studies will be needed to determine appropriate breakpoints before using CRP concentration as a clinical marker among cART initiators.

Interestingly, we did not confirm a significant association between the remaining immune activation biomarkers and early HIV progression on cART. While CD8+ T-cell activation has been associated with HIV disease progression, it has not been consistently associated with cART treatment outcomes $38-40,44-46$. Similarly, elevated baseline IL-6 levels, which have long been associated with HIV progression, has been an inconsistent predictor of outcomes among cART initiators $36,37,47,48$. IP-10, a chemokine that is strongly predictive of Hepatitis $\mathrm{C}$ virus treatment outcomes, has been associated with HIV outcomes in some studies 31,49 . We did find a trend for an association between $\mathrm{SCD} 14$ and clinical progression that confirms previous studies. Finally, the MT markers LPS and EndoCAb IgM have been associated with mortality and disease progression among cART initiators in some, but not all, studies $21,36,40,50$. Therefore, there may be a distinction between markers that predict disease progression in untreated patients, and markers that are predictive among HIVinfected patients receiving cART.

The present study did not provide evidence for MT in this cohort as defined by detectable LPS levels despite using an optimized assay that accounts for serum interference ${ }^{27}$. It is possible that prevalent diarrheal disease in some endemic settings may alter the associations of MT and HIV. However, sCD14 and CD8+ T-cell activation had a trend towards association with incident WHO Stage 3 or 4 disease or death on cART, which is consistent with and extends previous findings to multiple geographic settings. Overall, we speculate that a combination of factors, including socioeconomics, malnutrition, and prevalent acute infections (e.g., malaria, diarrheal disease), may have contributed to the generalized immune activation state of patients but that these factors may not have been directly involved in the causal pathways that led to HIV disease progression.

Strengths of the present study include the diversity of settings and the comprehensive panel of immune activation biomarkers assessed. However, this study has some potential limitations. Our study was limited to participants with CD4+ T cell counts $<300$ cells $/ \mathrm{mm}^{3}$; given current guidelines, future investigations will need to determine whether our findings are applicable to HIV-infected persons initiating cART at higher CD4+ T cell counts. There were a large number of prevalent and incident cases of TB, a leading cause of morbidity and mortality among HIV-infected persons in resource-limited settings. Therefore, it is possible that baseline, subclinical TB may have resulted in augmented immune activation, thus confounding the analysis. Notably, however, non-TB diagnoses comprised more than $70 \%$ of the incident diagnoses among cases; therefore, our findings are likely to be generalizable to HIV-infected populations, irrespective of TB risk. Moreover, our analysis accounted for 
the effect of prevalent tuberculosis at baseline, which did not substantially affect the overall strength of the predictive models. Notably, the entry criteria for the PEARLS study included a relatively healthy population. For example, fewer than $20 \%$ of cases and controls in the subcohort were underweight. Whereas on the one hand the relative health of the study population suggests that these findings will need confirmation, on the other hand we propose that an immune activation biomarker is particularly useful in identifying people where the risk of clinical progression is not obvious. Another challenge was the limited availability of viable PBMC samples for T-cell activation marker testing, especially from India and Thailand. Participants with available PBMC samples were similar to those without samples among baseline characteristics, except for lower median baseline HIV RNA level, which would be expected to underestimate the association between baseline CD4+ T-cell activation and HIV disease progression. Nevertheless, our findings will need to be confirmed in Asian populations to broaden the generalizability of CD4+ T-cell activation.

In summary, our analysis identified two biomarkers of immune activation that appear to independently predict early clinical HIV disease progression in multiple countries among HIV-infected adults initiating cART. While measurement of baseline CD4+/DR+/CD38+ Tcell activation may provide predictive information about the clinical response to cART, the utility of this biomarker may be limited due to its high cost and complexity in measuring it. CRP assays are low-cost, relatively easy to perform, and may be available in some resourcelimited settings. Pre-cART risk stratification using CRP measurements may prove worthwhile to identify patients for whom added vigilance, such as more frequent clinical monitoring or disease-specific screening after treatment initiation, may be warranted, similar to the cardiovascular disease paradigm.

\section{Supplementary Material}

Refer to Web version on PubMed Central for supplementary material.

\section{Acknowledgments}

The authors thank study coordinators and participants, and Veronique Franco and Sara Wendel for their assistance in performing microbial translocation assays. In addition, we thank Katherine McIntire for her editorial assistance.

Funding: This work was supported by the US National Institute of Allergy and Infectious Diseases [AI68636, AI069450, and 1 U01 AI068634 to the Statistical and Data Management Center of the AIDS Clinical Trials Group]; the US National Institutes of Health [R01 AI080417 to AG and R01 DA016078 to AB]; and additional grants from Boehringer-Ingelheim, Bristol-Myers Squibb, Gilead Sciences, and GlaxoSmithKline.

\section{References}

1. Larson HJ, Bertozzi S, Piot P. Redesigning the AIDS response for long-term impact. Bull World Health Organ. Nov 1; 2011 89(11):846-852. 2011. [PubMed: 22084531]

2. Gupta A, Nadkarni G, Yang WT, et al. Early mortality in adults initiating antiretroviral therapy (ART) in low- and middle-income countries (LMIC): a systematic review and meta-analysis. PLoS One. 2011; 6(12):e28691. [PubMed: 22220193]

3. Poka-Mayap V, Pefura-Yone EW, Kengne AP, Kuaban C. Mortality and its determinants among patients infected with HIV-1 on antiretroviral therapy in a referral centre in Yaounde, Cameroon: a retrospective cohort study. BMJ open. 2013; 3(7) 
4. Lapadula G, Cozzi-Lepri A, Marchetti G, et al. Risk of clinical progression among patients with immunological nonresponse despite virological suppression after combination antiretroviral treatment. Aids. Mar 13; 2013 27(5):769-779. [PubMed: 23719349]

5. Rodger AJ, Lodwick R, Schechter M, et al. Mortality in well controlled HIV in the continuous antiretroviral therapy arms of the SMART and ESPRIT trials compared with the general population. Aids. Mar 27; 2013 27(6):973-979. [PubMed: 23698063]

6. Antiretroviral Therapy Cohort C. Shepherd BS, Jenkins CA, et al. Higher rates of AIDS during the first year of antiretroviral therapy among migrants: the importance of tuberculosis. AIDS. May 15; 2013 27(8):1321-1329. [PubMed: 23925379]

7. May M, Sterne JA, Sabin C, et al. Prognosis of HIV-1-infected patients up to 5 years after initiation of HAART: collaborative analysis of prospective studies. AIDS. May 31; 2007 21(9):1185-1197. [PubMed: 17502729]

8. Boulle A, Schomaker M, May MT, et al. Mortality in Patients with HIV-1 Infection Starting Antiretroviral Therapy in South Africa, Europe, or North America: A Collaborative Analysis of Prospective Studies. PLoS medicine. Sep.2014 11(9):e1001718. [PubMed: 25203931]

9. Braitstein P, Brinkhof MW, Dabis F, et al. Mortality of HIV-1-infected patients in the first year of antiretroviral therapy: comparison between low-income and high-income countries. Lancet. Mar 11; 2006 367(9513):817-824. [PubMed: 16530575]

10. Chene G, Sterne JA, May M, et al. Prognostic importance of initial response in HIV-1 infected patients starting potent antiretroviral therapy: analysis of prospective studies. Lancet. Aug 30; 2003 362(9385):679-686. [PubMed: 12957089]

11. Bagchi S, Kempf MC, Westfall AO, Maherya A, Willig J, Saag MS. Can routine clinical markers be used longitudinally to monitor antiretroviral therapy success in resource-limited settings. Clin Infect Dis. Jan 1; 2007 44(1):135-138. [PubMed: 17143829]

12. Egger M, May M, Chene G, et al. Prognosis of HIV-1-infected patients starting highly active antiretroviral therapy: a collaborative analysis of prospective studies. Lancet. Jul 13; 2002 360(9327):119-129. 2002. [PubMed: 12126821]

13. Fregonese F, Collins IJ, Jourdain G, et al. Predictors of 5-year mortality in HIV-infected adults starting highly active antiretroviral therapy in Thailand. J Acquir Immune Defic Syndr. May 1; 2012 60(1):91-98. [PubMed: 22293548]

14. Helleberg M, Kronborg G, Larsen CS, et al. Poor CD4 response despite viral suppression is associated with increased non-AIDS-related mortality among HIV patients and their parents. Aids. Mar 27; 2013 27(6):1021-1026. [PubMed: 23196936]

15. Young J, Psichogiou M, Meyer L, et al. CD4 cell count and the risk of AIDS or death in HIVInfected adults on combination antiretroviral therapy with a suppressed viral load: a longitudinal cohort study from COHERE. PLoS medicine. 2012; 9(3):e1001194. [PubMed: 22448150]

16. Mocroft A, Furrer HJ, Miro JM, et al. The incidence of AIDS-defining illnesses at a current CD4 count $>/=200$ cells/muL in the post-combination antiretroviral therapy era. Clin Infect Dis. Oct; 2013 57(7):1038-1047. [PubMed: 23921881]

17. Sodora DL, Silvestri G. Immune activation and AIDS pathogenesis. Aids. Feb 19; 2008 22(4):439446. [PubMed: 18301056]

18. Brenchley JM, Price DA, Schacker TW, et al. Microbial translocation is a cause of systemic immune activation in chronic HIV infection. Nat Med. Dec; 2006 12(12):1365-1371. [PubMed: 17115046]

19. Ancuta P, Kamat A, Kunstman KJ, et al. Microbial translocation is associated with increased monocyte activation and dementia in AIDS patients. PLoS One. 2008; 3(6):e2516. [PubMed: 18575590]

20. Balagopal A, Philp FH, Astemborski J, et al. Human immunodeficiency virus-related microbial translocation and progression of hepatitis C. Gastroenterology. Jul; 2008 135(1):226-233. [PubMed: 18457674]

21. Sandler NG, Wand H, Roque A, et al. Plasma levels of soluble CD14 independently predict mortality in HIV infection. J Infect Dis. Mar 15; 2011 203(6):780-790. 2011. [PubMed: 21252259] 
22. Hunt PW, Martin JN, Sinclair E, et al. T cell activation is associated with lower CD4+ T cell gains in human immunodeficiency virus-infected patients with sustained viral suppression during antiretroviral therapy. J Infect Dis. May 15; 2003 187(10):1534-1543. 2003. [PubMed: 12721933]

23. Redd AD, Dabitao D, Bream JH, et al. Microbial translocation, the innate cytokine response, and HIV-1 disease progression in Africa. Proc Natl Acad Sci U S A. Apr 21; 2009 106(16):67186723. 2009. [PubMed: 19357303]

24. Campbell TB, Smeaton LM, Kumarasamy N, et al. Efficacy and safety of three antiretroviral regimens for initial treatment of HIV-1: a randomized clinical trial in diverse multinational settings. PLoS medicine. 2012; 9(8):e1001290. [PubMed: 22936892]

25. Barlow WE, Ichikawa L, Rosner D, Izumi S. Analysis of case-cohort designs. Journal of clinical epidemiology. Dec; 1999 52(12):1165-1172. [PubMed: 10580779]

26. Onland-Moret NC, van der AD, van der Schouw YT, et al. Analysis of case-cohort data: a comparison of different methods. Journal of clinical epidemiology. Apr; 2007 60(4):350-355. [PubMed: 17346608]

27. Balagopal A, Gama L, Franco V, et al. Detection of microbial translocation in HIV and SIV infection using the Limulus amebocyte lysate assay is masked by serum and plasma. PLoS One. 2012; 7(8):e41258. [PubMed: 22870212]

28. Grinsztejn B, Smeaton L, Barnett R, et al. Sex-associated differences in pre-antiretroviral therapy plasma HIV RNA in diverse areas of the world vary by CD4(+) T-cell count. Antivir Ther. 2011; 16(7):1057-1062. [PubMed: 22024521]

29. Pedersen KK, Pedersen M, Gaardbo JC, et al. Persisting inflammation and chronic immune activation but intact cognitive function in HIV-infected patients after long-term treatment with combination antiretroviral therapy. J Acquir Immune Defic Syndr. Jul 1; 2013 63(3):272-279. [PubMed: 23392469]

30. Carsenti-Dellamonica H, Saidi H, Ticchioni M, et al. The suppression of immune activation during enfuvirtide-based salvage therapy is associated with reduced CCR5 expression and decreased concentrations of circulating interleukin-12 and IP-10 during 48 weeks of longitudinal follow-up. HIV Med. Feb; 2011 12(2):65-77. [PubMed: 20500532]

31. Jiao Y, Zhang T, Wang R, et al. Plasma IP-10 is associated with rapid disease progression in early HIV-1 infection. Viral Immunol. Aug; 2012 25(4):333-337. 2012. [PubMed: 22788418]

32. Duong T, Jourdain G, Ngo-Giang-Huong N, et al. Laboratory and clinical predictors of disease progression following initiation of combination therapy in HIV-infected adults in Thailand. PLoS One. 2012; 7(8):e43375. [PubMed: 22905264]

33. Smith CJ, Ryom L, Weber R, et al. Trends in underlying causes of death in people with HIV from 1999 to 2011 (D:A:D): a multicohort collaboration. Lancet. Jul 19; 2014 384(9939):241-248. [PubMed: 25042234]

34. Drain PK, Kupka R, Msamanga GI, Urassa W, Mugusi F, Fawzi WW. C-reactive protein independently predicts HIV-related outcomes among women and children in a resource-poor setting. Aids. Oct 1; 2007 21(15):2067-2075. [PubMed: 17885297]

35. Redd AD, Eaton KP, Kong X, et al. C-reactive protein levels increase during HIV-1 disease progression in Rakai, Uganda, despite the absence of microbial translocation. J Acquir Immune Defic Syndr. Aug; 2010 54(5):556-559. 2010. [PubMed: 20463585]

36. Boulware DR, Hullsiek KH, Puronen CE, et al. Higher levels of CRP, D-dimer, IL-6, and hyaluronic acid before initiation of antiretroviral therapy (ART) are associated with increased risk of AIDS or death. J Infect Dis. Jun 1; 2011 203(11):1637-1646. [PubMed: 21592994]

37. Kuller LH, Tracy R, Belloso W, et al. Inflammatory and coagulation biomarkers and mortality in patients with HIV infection. PLoS medicine. Oct 21.2008 5(10):e203. [PubMed: 18942885]

38. Rudy BJ, Lindsey JC, Flynn PM, et al. Immune reconstitution and predictors of virologic failure in adolescents infected through risk behaviors and initiating HAART: week 60 results from the PACTG 381 cohort. AIDS research and human retroviruses. Mar; 2006 22(3):213-221. [PubMed: 16545007]

39. Hunt PW, Cao HL, Muzoora C, et al. Impact of CD8+ T-cell activation on CD4+ T-cell recovery and mortality in HIV-infected Ugandans initiating antiretroviral therapy. Aids. Nov 13; 2011 25(17):2123-2131. [PubMed: 21881481] 
40. Taiwo B, Matining RM, Zheng L, et al. Associations of T cell activation and inflammatory biomarkers with virological response to darunavir/ritonavir plus raltegravir therapy. The Journal of antimicrobial chemotherapy. Aug; 2013 68(8):1857-1861. [PubMed: 23599363]

41. Roine I, Arguedas A, Faingezicht I, Rodriguez F. Early detection of sequela-prone osteomyelitis in children with use of simple clinical and laboratory criteria. Clin Infect Dis. May; 1997 24(5):849853. [PubMed: 9142781]

42. Mora S, Ridker PM. Justification for the Use of Statins in Primary Prevention: an Intervention Trial Evaluating Rosuvastatin (JUPITER)--can C-reactive protein be used to target statin therapy in primary prevention. The American journal of cardiology. Jan 16; 2006 97(2a):33a-41a.

43. Pearson TA, Mensah GA, Alexander RW, et al. Markers of inflammation and cardiovascular disease: application to clinical and public health practice: A statement for healthcare professionals from the Centers for Disease Control and Prevention and the American Heart Association. Circulation. Jan 28; 2003 107(3):499-511. [PubMed: 12551878]

44. Mildvan D, Bosch RJ, Kim RS, et al. Immunophenotypic markers and antiretroviral therapy (IMART): T cell activation and maturation help predict treatment response. J Infect Dis. May 15; 2004 189(10):1811-1820. [PubMed: 15122517]

45. Ondoa P, Koblavi-Deme S, Borget MY, Nolan ML, Nkengasong JN, Kestens L. Assessment of CD8 T cell immune activation markers to monitor response to antiretroviral therapy among HIV-1 infected patients in Cote d'Ivoire. Clin Exp Immunol. Apr; 2005 140(1):138-148. [PubMed: 15762885]

46. Zhang JC, Zhang HJ, Li Y, et al. Changes in levels of T cell subpopulations to monitor the response to antiretroviral therapy among HIV-1-infected patients during two years of HIV-1 replication suppression. Scand J Infect Dis. May; 2013 45(5):368-377. [PubMed: 23186319]

47. Kalayjian RC, Machekano RN, Rizk N, et al. Pretreatment levels of soluble cellular receptors and interleukin-6 are associated with HIV disease progression in subjects treated with highly active antiretroviral therapy. J Infect Dis. Jun 15; 2010 201(12):1796-1805. [PubMed: 20446847]

48. Rodger AJ, Fox Z, Lundgren JD, et al. Activation and coagulation biomarkers are independent predictors of the development of opportunistic disease in patients with HIV infection. J Infect Dis. Sep 15; 2009 200(6):973-983. [PubMed: 19678756]

49. Zeremski M, Markatou M, Brown QB, Dorante G, Cunningham-Rundles S, Talal AH. Interferon gamma-inducible protein 10: a predictive marker of successful treatment response in hepatitis C virus/HIV-coinfected patients. J Acquir Immune Defic Syndr. Jul 1; 2007 45(3):262-268. 2007. [PubMed: 17414926]

50. Marchetti G, Cozzi-Lepri A, Merlini E, et al. Microbial translocation predicts disease progression of HIV-infected antiretroviral-naive patients with high CD4+ cell count. Aids. Jul 17; 2011 25(11):1385-1394. [PubMed: 21505312] 


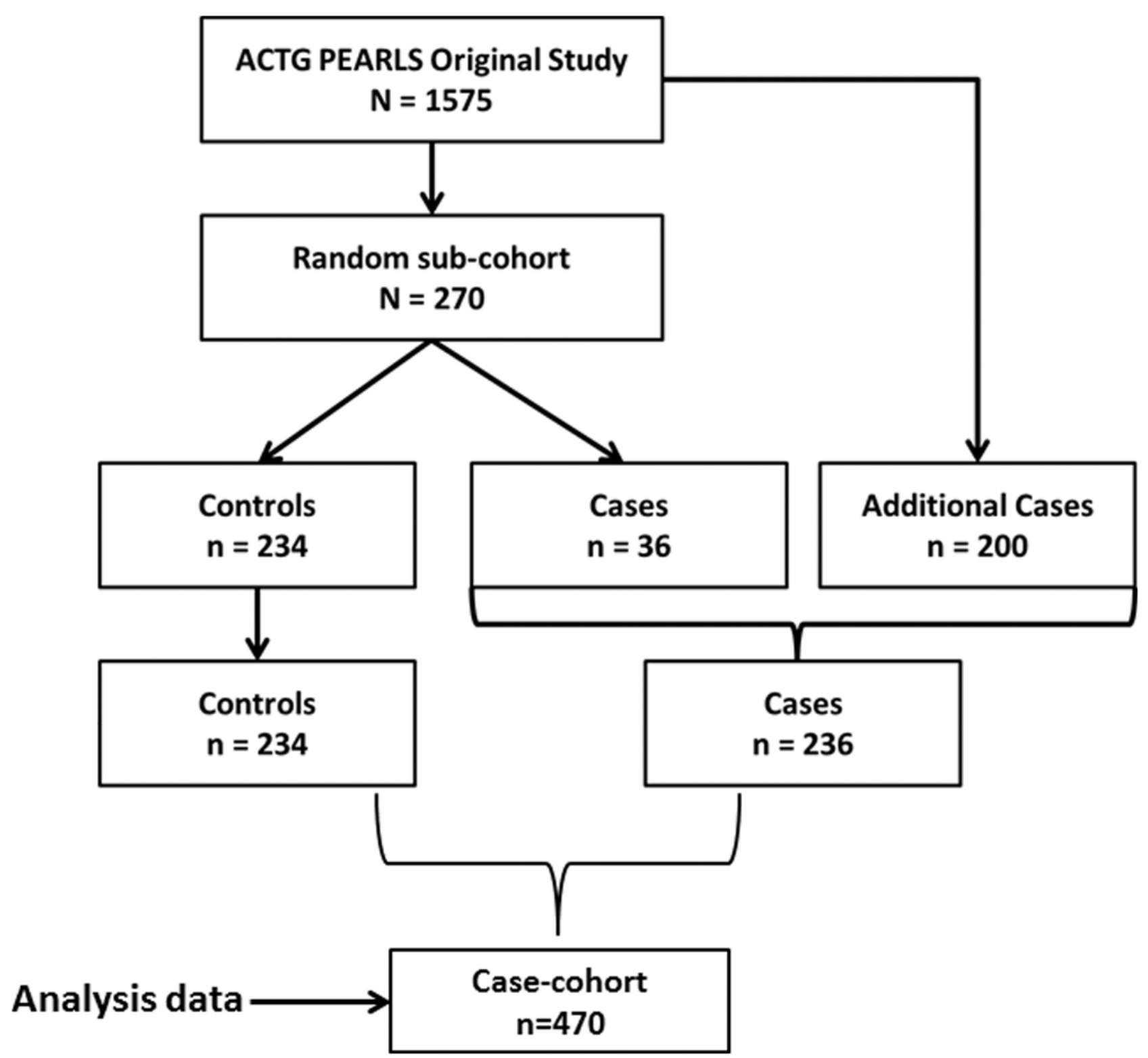

Figure 1. Formation of the case-cohort

The case-cohort analysis $(n=470)$ consisted of the random subcohort (a randomly selected sub-sample of 30 participants per country [n=270]) from the original ACTG PEARLS study and any additional cases meeting our case definition from the original study cohort [n=200]. Cases were defined as incident WHO stage 3 or 4 event or death by 96 weeks post-cART initiation ( $\mathrm{n}=236)$. Immune-activation biomarkers were quantified pre-cART in the random subcohort to estimate baseline immune activation in the original study cohort. Adjusted multivariate models estimated associations between individual pre-cART biomarker values and the primary outcome, incident WHO stage 3 or 4 event or death by 96 weeks on cART. 


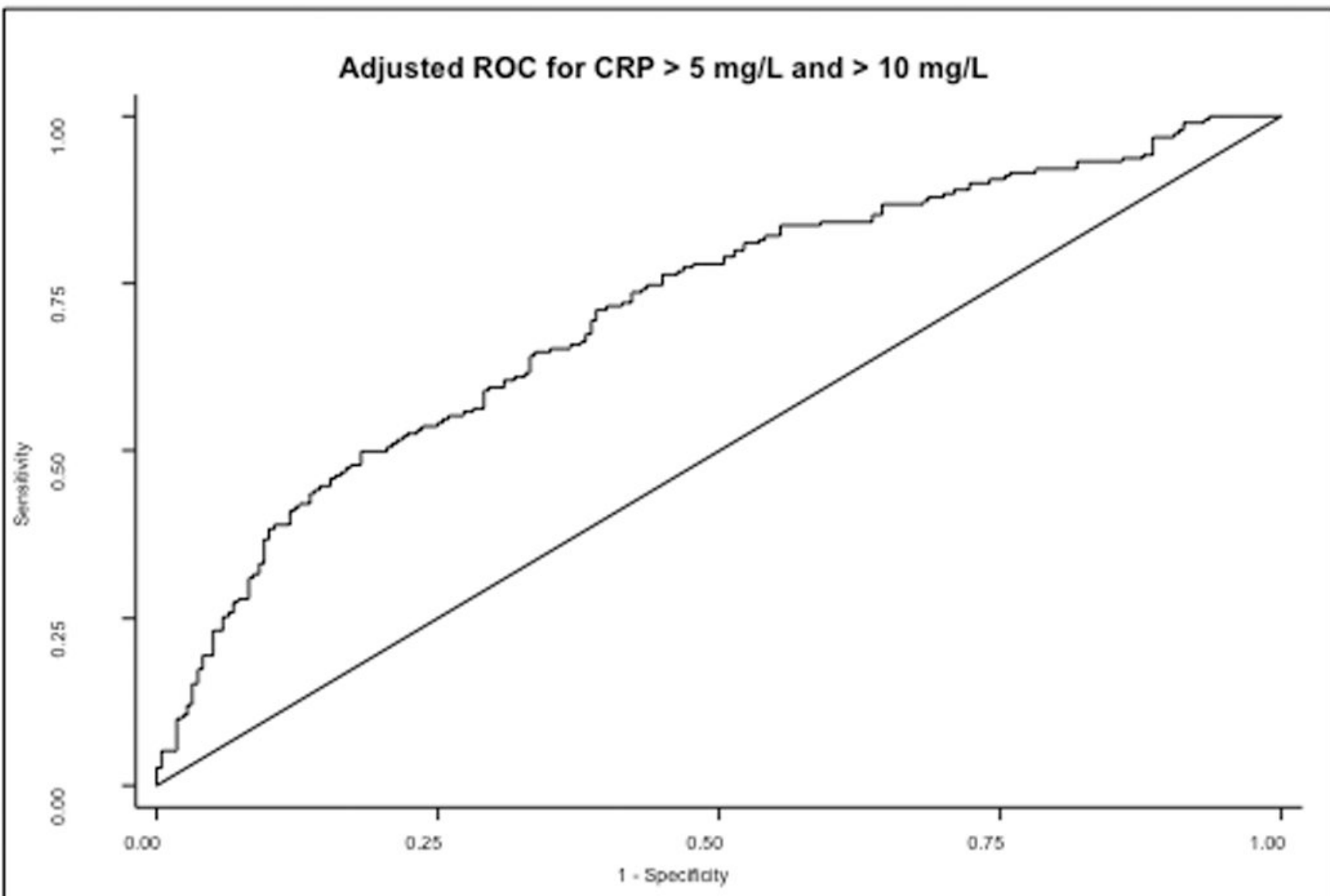

AUC (95\% Cl): $0.71(95 \% \mathrm{Cl} 0.66,0.76)$

\section{$\mathrm{AUC}=0.71$}

Figure 2. Pre-cART CRP as a prediction tool for clinical failure

ROC analysis was performed to evaluate if elevated levels of CRP pre-cART can be used to predict clinical failure post-cART initiation. The CRP cutoff was varied using observed CRP levels. After adjusting for baseline age, sex, country, BMI, hemoglobin, CD4+ T-cell count, plasma HIV RNA, and baseline TB, the area under the curve (AUC) was 0.71, irrespective of the cutoff used (CRP>5 mg/L or CRP>10 mg/L). 
Table 1

Baseline characteristics of the case-cohort by study group ${ }^{a}$

\begin{tabular}{|c|c|c|c|c|}
\hline Characteristic & Overall $(n=470)$ & $\operatorname{Cases}^{b}(n=236)$ & Controls (n=234) & $P$ \\
\hline Age, median (IQR), y & $35(29-40)$ & $35(28-40)$ & $35(29-41)$ & \\
\hline Female & $215(45)$ & $100(42)$ & $115(49)$ & 0.22 \\
\hline \multicolumn{5}{|l|}{ DAIDS Race } \\
\hline White & $48(9)$ & $20(8)$ & $28(12)$ & \\
\hline Black & $231(49)$ & $107(45)$ & $124(53)$ & \\
\hline Asian & $146(31)$ & $97(41)$ & $49(21)$ & 0.003 \\
\hline Hispanic & $0(0)$ & $0(0)$ & $0(0)$ & \\
\hline Other & $45(10)$ & $12(5)$ & $33(14)$ & \\
\hline \multicolumn{5}{|l|}{ Country } \\
\hline Brazil & $45(10)$ & $16(7)$ & $29(12)$ & \\
\hline Haiti & $38(8)$ & $13(6)$ & $25(11)$ & \\
\hline India & $107(23)$ & $87(37)$ & $20(9)$ & \\
\hline Malawi & $62(13)$ & $38(16)$ & $24(10)$ & \\
\hline Peru & $36(8)$ & $8(3)$ & $28(12)$ & 0.005 \\
\hline S. Africa & $58(12)$ & $33(14)$ & $25(11)$ & \\
\hline Thailand & $37(8)$ & $9(4)$ & $28(12)$ & \\
\hline USA & $49(10)$ & $20(8)$ & $29(12)$ & \\
\hline Zimbabwe & $38(8)$ & $12(5)$ & $26(11)$ & \\
\hline \multicolumn{5}{|l|}{ BMI, $\mathrm{kg} / \mathrm{m}^{2}$} \\
\hline Underweight $(<18.5)$ & $58(12)$ & $38(16)$ & $20(9)$ & \\
\hline Normal $(18.5$ to $<25)$ & $315(67)$ & $163(69)$ & $152(65)$ & 0.0001 \\
\hline Overweight $(25$ to $<30)$ & $74(16)$ & $27(11)$ & $47(20)$ & \\
\hline Obese $(\geq 30)$ & $23(5)$ & $8(3)$ & $15(6)$ & \\
\hline \multicolumn{5}{|c|}{ CD4+ T-cell count, cells $/ \mathbf{m m}^{3}$} \\
\hline$<50$ & $83(18)$ & $46(19)$ & $37(16)$ & \\
\hline $50-99$ & $59(13)$ & $33(14)$ & $26(11)$ & \\
\hline $100-199$ & $162(34)$ & $85(36)$ & $77(33)$ & 0.004 \\
\hline $200-249$ & $97(21)$ & $48(20)$ & $49(21)$ & \\
\hline $250-299$ & $69(15)$ & $24(10)$ & $45(19)$ & \\
\hline \multicolumn{5}{|l|}{ HIV RNA, cp/mL } \\
\hline$<400$ & $4(1)$ & $1(0.4)$ & $3(1)$ & \\
\hline 400 to $<4000$ & $16(3)$ & $6(3)$ & $10(4)$ & \\
\hline 4000 to $<40000$ & $98(21)$ & $48(20)$ & $50(21)$ & 0.06 \\
\hline 40000 to $<400000$ & $254(54)$ & $123(52)$ & $131(56)$ & \\
\hline$\geq 400000$ & $98(21)$ & $58(25)$ & $40(17)$ & \\
\hline TB prevalence & $112(24 \%)$ & $75(32 \%)$ & $37(16 \%)$ & 0.001 \\
\hline HBsAg prevalence & $24(5 \%)$ & $11(5 \%)$ & $13(5 \%)$ & 0.97 \\
\hline \multicolumn{5}{|l|}{ Treatment Regimen } \\
\hline 3TC-ZDV + EFV & $171(36)$ & $90(38)$ & $81(35)$ & \\
\hline
\end{tabular}




\begin{tabular}{lcccc}
\hline Characteristic & Overall $(\mathbf{n}=\mathbf{4 7 0})$ & Cases $^{\boldsymbol{b}}(\mathbf{n = 2 3 6})$ & Controls $(\mathbf{n}=\mathbf{2 3 4})$ & $\boldsymbol{P}$ \\
\hline DDI + FTC + ATV & $149(32)$ & $71(30)$ & $78(33)$ & 0.81 \\
TDF-FTC + EFV & $150(32)$ & $75(32)$ & $75(32)$ & \\
\hline
\end{tabular}

Abbreviations: ATV, atazanavir; BMI, body mass index; DAIDS, Division of AIDS; DDI, didanosine; EFV, efavirenz; FTC, emtricitabine; $\mathrm{HBsAg}$, hepatitis B surface antigen; IQR, interquartile range; TB, tuberculosis; TDF, tenofovir; ZDV, zidovudine; 3TC, lamivudine.

${ }^{a}$ Data presented as n (\%) unless otherwise indicated.

${ }^{b}$ Cases defined as any incident WHO stage 3 or 4 event or death by 96 weeks post cART initiation. 
Table 2

Pre-cART immune-activation biomarker values and number tested $(\mathrm{N})$ in the random subcohort $(n=270)$ and by study group in the case-cohort $(n=470)$

\begin{tabular}{|c|c|c|c|c|}
\hline \multirow[t]{2}{*}{ Biomarker $^{a}$} & \multirow{2}{*}{$\underset{(\mathrm{n}=270)}{\text { Random Subcohort }^{e}}$} & \multicolumn{3}{|c|}{ Case-Cohort $f$} \\
\hline & & $\operatorname{Case}^{b}(n=236)$ & Control $(n=234)$ & $P^{c}$ \\
\hline Detectable $\mathrm{LPS}^{d}, \mathrm{n}(\%)$ & $92(41)$ & $55(32)$ & $82(42)$ & 0.06 \\
\hline $\mathrm{sCD} 14, \mathrm{pg} / \mathrm{mL}$ & $2.1 \times 10^{6}\left(5.53 \times 10^{5}-2.7 \times 10^{6}\right)$ & $2.1 \times 10^{6}\left(3.49 \times 10^{5}-2.9 \times 10^{6}\right)$ & $2.0 \times 10^{6}\left(5.11 \times 10^{5}-2.7 \times 10^{6}\right)$ & 0.81 \\
\hline EndoCAb IgM, MMU/mL & $48.08(29.7-71.5)$ & $44.28(27.3-67.7)$ & $49.43(29.7-69.7)$ & 0.30 \\
\hline $\mathrm{IFN} \gamma, \mathrm{pg} / \mathrm{mL}$ & $17.47(6.12-49.65)$ & $15.88(4.99-41.45)$ & $17(6.04-55.14)$ & 0.78 \\
\hline $\mathrm{IL}-6, \mathrm{pg} / \mathrm{mL}$ & $23.76(8.89-51.04)$ & $27.47(11.02-49.62)$ & $24.01(8.89-50.34)$ & 0.45 \\
\hline $\mathrm{TNFa}, \mathrm{pg} / \mathrm{mL}$ & $19.13(13.19-26.67)$ & $20.4(14.11-33.79)$ & $19.13(13.35-26.67)$ & 0.60 \\
\hline $\mathrm{IP}-10, \mathrm{pg} / \mathrm{mL}$ & $1228.67(584.53-2665.64)$ & $2079.11(701.73-4043.64)$ & $1203.81(584.53-2665.64)$ & 0.02 \\
\hline $\mathrm{CRP}, \mathrm{mg} / \mathrm{L}$ & $3.36(1.37-10.27)$ & $6.3(1.85-20.58)$ & $3.03(1.23-9.29)$ & 0.001 \\
\hline $\mathrm{CD} 8+/ \mathrm{DR}+/ 38+, \% \mathrm{CD} 8$ & $45.73(34.76-56.01)$ & $46.97(37.45-60.17)$ & $45.79(34.76-56.01)$ & 0.70 \\
\hline $\mathrm{CD} 4+/ \mathrm{DR}+/ 38+, \% \mathrm{CD} 4$ & $22.60(15.28-34.78)$ & $28.63(14.72-42.28)$ & $22.29(15.28-33.82)$ & 0.33 \\
\hline
\end{tabular}

Abbreviations: CRP, C-reactive protein; LPS, Gram-negative bacterial lipopolysaccharide, N, number of samples tested.

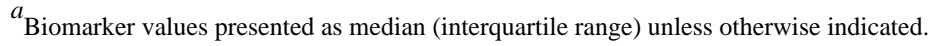

${ }^{b}$ Case defined as incident WHO stage 3 or 4 event or death by 96 weeks post cART initiation.

${ }^{c}$ P-values shown are for comparisons of cases and controls.

$d_{\text {LPS measurement was dichotomized as detectable vs. undetectable due to low detection rate among samples (137/367) despite using an optimized }}$ assay [16].

$e^{e}$ Between 241-255 samples were available for testing of soluble markers; 102 viable PBMC samples were available for testing of cellular markers.

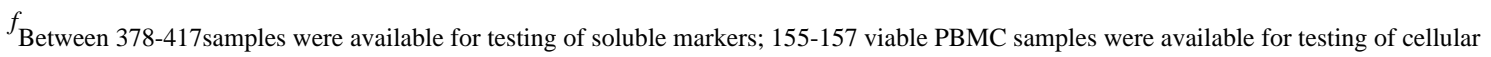
markers. 


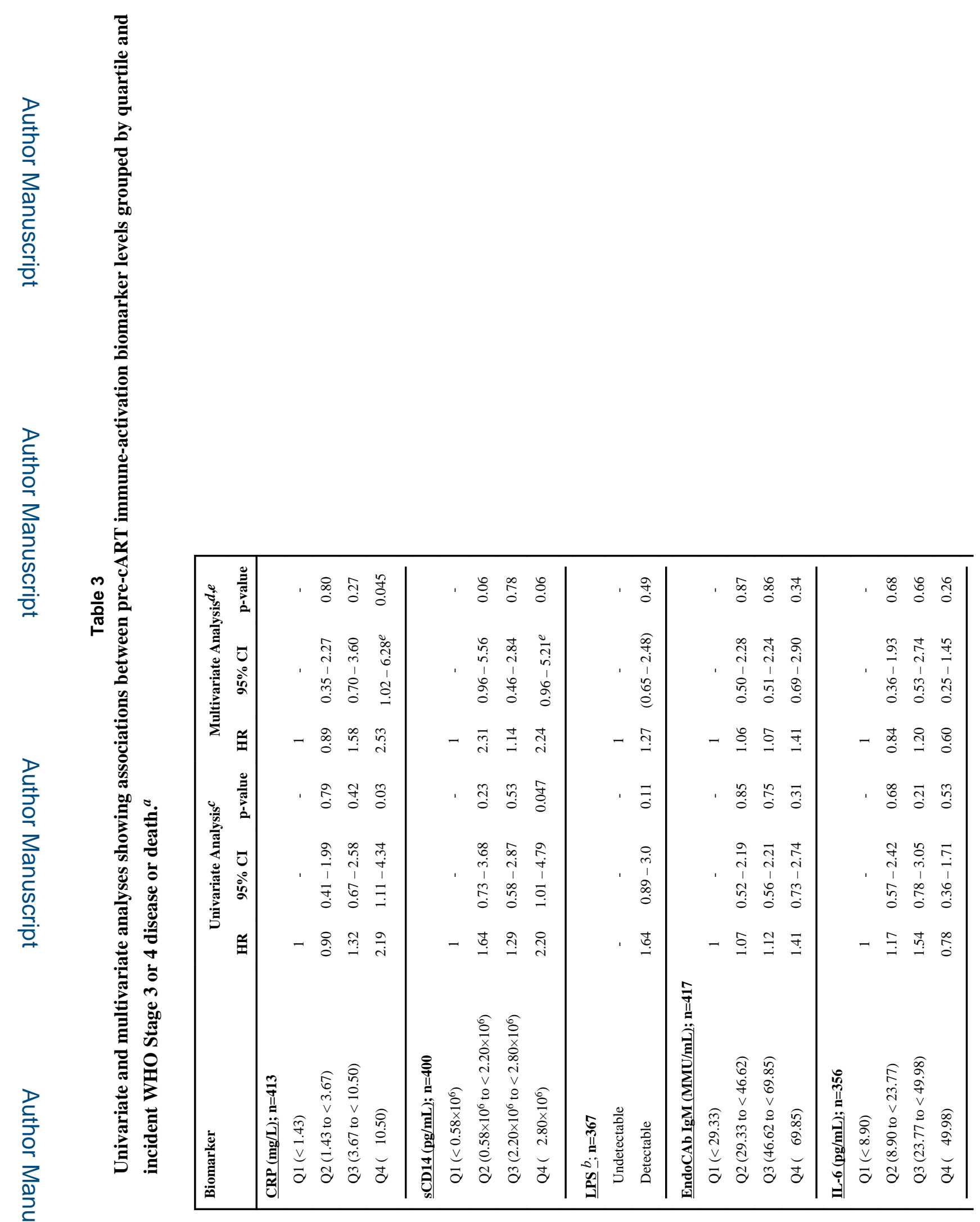

J Acquir Immune Defic Syndr. Author manuscript; available in PMC 2016 October 01. 


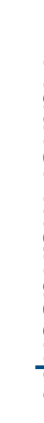

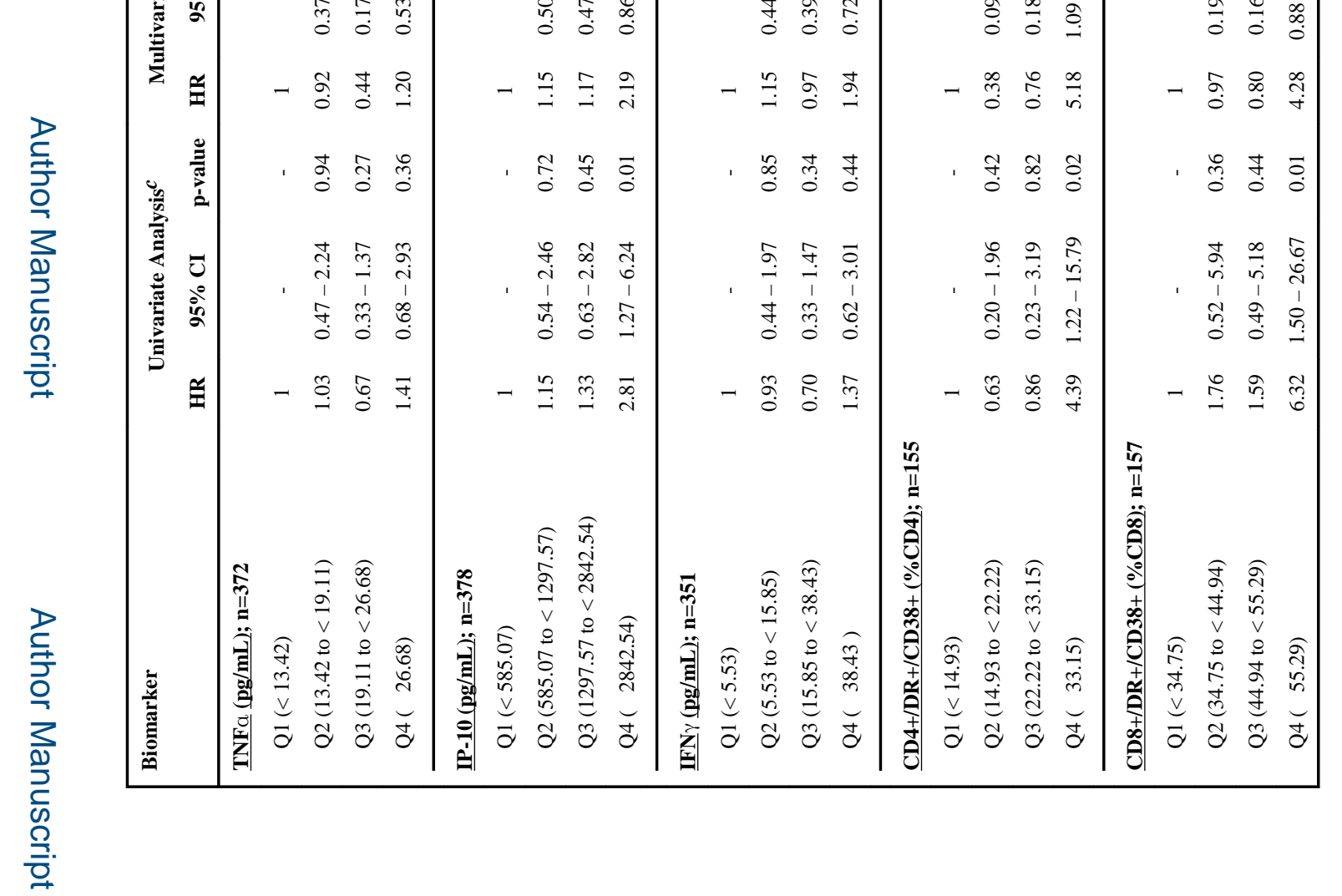

J Acquir Immune Defic Syndr. Author manuscript; available in PMC 2016 October 01. 


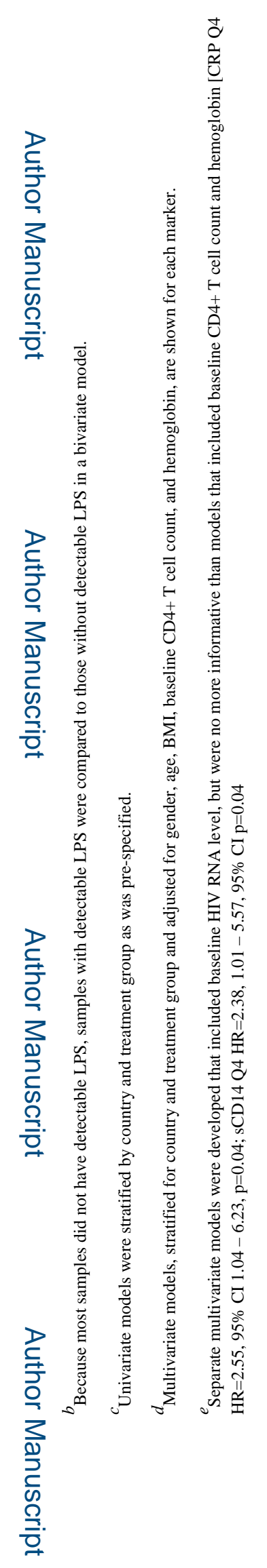

